\title{
An Agent-based Route Choice Model
}

\author{
Shanjiang Zhu* David Levinson* $\quad$ Lei Zhang ${ }^{\dagger}$
}

April 28, 2007

\begin{abstract}
Travel demand emerges from individual decisions. These decisions, depending on individual objectives, preferences, experiences and spatial knowledge about travel, are both heterogeneous and evolutionary. Research emerging from fields such as road pricing and ATIS requires travel demand models that are able to consider travelers with distinct attributes (value of time (VOT), willingness to pay, travel budgets, etc.) and behavioral preferences (e.g. willingness to switch routes with potential savings) in a differentiated market (by tolls and the level of service). Traditional trip-based models have difficulty in dealing with the aforementioned heterogeneity and issues such as equity. Moreover, the role of spatial information, which has significant influence on decision-making and travel behavior, has not been fully addressed in existing models. To bridge the gap, this paper proposes to explicitly model the formation and spreading of spatial knowledge among travelers. An Agent-based Route Choice (ARC) model was developed to track choices of each decision-maker on a road network over time and map individual choices into macroscopic flow pattern. ARC has been applied on both SiouxFalls network and
\end{abstract}

${ }^{*}$ University of Minnesota, Department of Civil Engineering, 500 Pillsbury Drive SE, Minneapolis, MN 55455 USA

${ }^{\dagger}$ Department of Civil, Construction, and Environmental Engineering Oregon State University, 220 Owen Hall, Corvallis, OR 97331 
Chicago sketch network. Comparison between ARC and existing models (UE and SUE) on both networks shows ARC is valid and computationally tractable. To be brief, this paper specifically focuses on the route choice behavior, while the proposed model can be extended to other modules of travel demand under an integrated framework.

Keywords: Agent-based model, route choice, traffic assignment, travel demand modeling

\section{Introduction}

Travel demand emerges from individual decisions. Travelers make decisions according to their individual objectives, preferences, experiences and knowledge about travel. Furthermore, previous decisions provide travelers with unique experience and spatial knowledge, thus influencing their subsequent decisions. Therefore, travelers decisions are both heterogeneous and evolutionary (Levinson, 1995; Srinivasan and Mahmassani, 2003; McFadden, 2002; Yang and Huang, 2004). There has been long-established effort both by practitioners and researchers to bridge the gap by mapping individual travel decisions onto a macroscopic context. Because of the behavioral nature of travel decisions, it is widely believed that travel demand models should treat individual traveler as the basic units of analysis based on solid behavioral principals (Ramming, 2002; Balmer et al., 2004; Zhang and Levinson, 2004; Zhang, 2006). Mainstream research and practice, however, has treated trips as the units of analysis since the 1950s due to the lack of deep understanding of the mechanisms of travel behavior and limitation in data, computational power, and algorithms. By assuming homogeneity, these models simplify the object of study to analyze large networks. Consequently, these models received great popularity in practice during the sec-

ond half of the twentieth century and many algorithms and commercial software 
packages have been developed to implement these aggregate models.

However, this trip based modeling paradigm encounters great difficulty when it tries to describe and evaluate some emerging initiatives to mitigate congestion and pollution, such as toll roads and advanced traveler information systems. For example, toll roads differentiate level of service on the supply side. As travelers who have distinct attributes (value of time (VOT), willingness to pay, travel budgets, etc.) and behavioral preferences (e.g. willingness to switch routes with potential savings) adjust their travel decisions in a differentiated market, the network can exhibit new traffic patterns, which will further affect pricing strategies and investment decisions. Traditional trip based models cannot provide an accurate description of this complex mechanism, as they are incapable of addressing important issues such as equity. Actually, many researchers (Estache and Strong, 2000; Yang and Zhang, 2002; Santos and Rojey, 2004) have emphasized the importance of equity as a consequence of road pricing and pointed out equity is an individual, not a group, problem (Evans, 1992). To account for this complexity, transportation economists and policy makers have long advanced their focus from first-best prices with homogeneous network assumptions to second-best prices under heterogeneity in both network users and service providers (Pigou, 1920; Knight, 1924; Mohring and Harwitz, 1962; Vickrey, 1963; Button and Verhoef, 1998; Levinson, 2005; Zou and Levinson, 2006), which requires explicitly modeling individual travelers route choice behavior. Although some researchers have studied these problems on small networks, a behavior-based model, which is not only sufficiently accurate but also applicable on large network, does not exist (Zhang, 2006).

It is crucial to recognize that travel decisions are based on travelers' knowledge about the network. Travelers, limited in their capability of acquiring, processing and storing spatial knowledge, can only consider the routes they know. 
This is the very reason that engineers try to assist travelers to make decisions by providing additional information about the network through technological innovations such as ATIS. As indicated by Srinivasan and Mahmassani (2003), ATIS cannot be well evaluated without explicitly accounting for the heterogeneity in behavior and the presence of dynamic learning and adjustment processes in user behavior. Many other researchers (Tversky and Simonson, 1993; Zhang et al., 2004) also emphasized the role of information and learning in traveler behavior such as route choice decisions. However, limited work has been done to systematically model the mechanism of acquiring, processing, storing of spatial knowledge and its communication among travelers.

To bridge the gap between the aforementioned demand and current practice in modeling, this paper explicitly models the formation and spreading of spatial knowledge (route information) among travelers and tracks the choices of each decision-maker on a road network over time. Individual travelers are treated as the unit of analysis and extending previous work (Zhang and Levinson, 2004), an Agent-based Route Choice (ARC) model is built to map individual choices into system flow patterns. Given a set of system parameters, the modeled road network can evolve a specific flow pattern, replicating real-world network changes. This agent-based approach, representing a significant departure from the previous literature that has primarily relied on equilibrium analysis, is also applicable on large real-world transportation networks. The second section reviews previous research, and introduces the new ARC model. Its properties are discussed and application on the Chicago Sketch network is demonstrated. Models such as ARC produce insights that may have significant implications for a more comprehensive agent-based travel demand model. 


\section{Literature Review}

Route choice model maps travel demand (often defined by an origin-destination (OD) trip table) into a link flow pattern, which is also known as a traffic assignment. Theoretical research in this field has a long history. Varying in assumptions of route choice behavior, many assignment models and corresponding algorithms have been proposed. All-or-Nothing is a simple route choice model that assumes travelers minimize some disutility (usually travel time) while ignoring what other travelers are doing. If we take other travelers into account, this minimization assumption, known as Wardrop's first principle (Wardrop, 1952), leads to the User Equilibrium model. The original UE models assumed perfect knowledge of the network by travelers and also assumed all travelers were identical (homogeneous). To account for the heterogeneity problem, researchers introduced the bi-criteria or multiclass assignment problem (Arnott and Kraus, 1998; Yang and Huang, 2004), in which travelers are differentiated into groups and characteristics such as VOT are identical within group and different between groups.

Other researchers tried to solve heterogeneity problem employing discrete choice models. Introducing a random perception error in travel time and treating it with Multinomial Logit model, Daganzo and Sheffi (1977) proposed the Stochastic User Equilibrium model. This model suffers from the so-called Independence of Irrelevant Alternative problem and many alternatives, including Nested Logit, Probit and Logit Kernel model, have been proposed to mitigate this problem (Ramming, 2002). However, these models only model how to choose route among alternatives but tell little about how to generate the route choice set. To generate the route choice set, sometimes known as the K-Shortest Path problem, several solutions based on Dijkstras algorithm (Ziliaskopoulos, 1994) have been introduced. Other heuristic approaches such as 
Link Elimination Approaches by Azevedo et al. (1993), Link Penalty Approaches by De La Barra et al. (1993), and Branching approach by Bellman and Kalaba (1960), have also been used. These approaches are also related to shortest path algorithm and lack an explicit behavioral foundation.

As computer simulation becomes more and more common in transportation, researchers are now able to model and simulate individuals traveling on large networks. As a result, simulation models are increasingly common in the field of route choice study because of their capability of modeling and tracking behavior of individual travelers over time. Mahmassani and Hawas (1998) developed DYNASmart, which simulates not only day-to-day but also on-road route choice decisions. DYNASmart focuses more on modeling travelers choice given the presence of additional information rather than how such information is generated. Agent-based modeling has received increasing emphasis in transportation (Transportation Research Part C (2002 Volume: 10C 325-527) has dedicated a special issue to this topic) because of its capability in modeling individual components and their interactions in complex network. Following this modeling strategy, Nagel and Barrett (1999); Nagel and Rickert (2001) proposed TRANSIMS, assuming travelers take the shortest path constrained by activity choices and generating flow patterns through Cellular Automata simulation. Noticing the limitation of the shortest path assumption, researchers (Balmer et al., 2004; Charypar and Nagel, 2005; Balmer and Nagel, 2006) proposed Matsim as an extension, in which choice sets are generated by randomly mutating routes and then discarding unrealistic options (e.g. routes with unreasonably high cost or using modes inconsistent with traveler characteristics). While this algorithm is enlightening and capable of dealing with multi-modal cases, the route choice set generation is inefficient and unrealistic.

Though many heuristic algorithms of route choice set generation have been 
proposed, few questions have been asked about how spatial knowledge (specifically route knowledge) is generated and spread among travelers. Studies in behavioral geography (Golledge and R.J., 1997), including both place learning theories and developmental theories, may shed light on this issue. The former hypothesized individuals learn and store routes as relationships of locations, while the latter argues route knowledge is acquired through experience and stored hierarchically according to their importance (people are more likely to know routes near their home, work place and frequently visited locations) and salience (freeways are more easily memorized). In recognition of the large amount of spatial information and numerous evidence of the distortion and incompleteness of stored spatial knowledge, Wolpert (1952) argues that spatial knowledge is boundedly rational rather than utility maximizing. Informationprocessing theories of choices also suggests decision makers choice strategies are simple, local and myopic when facing complex system (Olshavsky, 1979; Payne and Johnson, 1993; de Palma et al., 1994). Moreover, Bandura (1977)'s learning theory suggested that travelers can learn routes from each other through observing or communicating. And route learning could be trial-and-error, characterized by evolutionary development of knowledge and adjustments in behavior over time. A comprehensive review of spatial learning theory has been provided by Zhang (2006).

\section{Agent-Based Travel Demand Model}

\subsection{Model framework}

As presented, spatial learning theory suggests 1) travelers learn routes through experiencing and communicating with others, 2) spatial knowledge has hierarchy and can be communicated, and 3) travelers capability of processing spatial 
knowledge is bounded and a trial-and-error process may be involved. Based on these assumptions, together with the assumptions of heterogeneous travelers, this section introduces the Agent-based Route Choice (ARC) model.

ARC simulates spatial learning, tracks evolutionary route choices, and generates macroscopic flow patterns, which may be used to evaluate policy initiatives or used as input for a more comprehensive travel demand model. Travelers form spatial information through traveling and experiencing, and then spread that information on the network through communication. They also make decisions about destinations, time schedule, mode and travel route, all of which comprise the demand side of travel activities (in this paper, to avoid confounding factors, all but route are taken as given). Nevertheless, these demands are constrained by the supply: network capacity and tolls, which are also taken as given in this paper. All the decision makers have their individual attributes, goals and behavior rules, and will make individual decisions based on information gained through exploring the network or social exchange of information. Integrated with other agent-based models, including residential and job location choice, land use, and infrastructure investment models, ARC could enable a wide spectrum of studies in transportation and land use related topics. The following paragraphs describe the modeling framework in detail.

\subsection{Agent}

The model contains three types of interactive agents: travelers, nodes, and links, all of which have their individual attributes, goals and rules.

Travelers are characterized by their willingness to pay $(\mathrm{u})$, value of time $(\mathrm{v})$ and capability of getting information (s). Willingness to pay reflects how many resources have been allocated to travel and thus determines how far a traveler could travel. Individual value of time provides travelers impetus to choose routes 
with different travel time and toll combinations. Travelers with a larger social network and better information resources distinguish themselves in their ability of getting information from their peers who have a less effective social network and less exposure to new information. Therefore, travelers learn information about network through both experience and learning from other travelers and choose routes based on the information acquired and individual characteristics.

Consistent with current practice in planning, there are two categories of nodes: network vertices and centroids. While vertices represent starting and ending point of links, centroids represent residential locations and workplaces. Applying social learning theory, centroids are also where people interact with each other and communicate spatial information. Therefore, centroids also stand for the neighborhood where travel information is pooled and communicated. For example, people can learn travel information from their colleagues at the workplace or from neighbors meeting in a grocery store. Suggested by spatial learning theory, traffic information can only be generated through experience. However, information brought to centroids will be pooled and shared by all frequent visitors (e.g. people live or work here). Limited by information processing capability, only up to K shortest paths from every other node on the network to the current node will be kept. This information is updated as new information is available and fed back to subsequent visiting travelers.

Links represent directed roads and are labeled with the origin, destination, capacity $(\mathrm{C})$, free flow travel time (to), and toll rate (p). Link travel time (t) and generalized link cost (c) can be derived given the traffic flow and link cost function. The BPR function is employed in the model to map flows to travel time. 


\subsection{Rules}

ARC simulates individual route choices and determines the flow pattern on the network subject to a given OD distribution.

The initial route choice can be either given or generated by a random-walk route searching process at iteration 0 . In the random walk scenario, travelers set off from their origins and travel in a randomly chosen direction after arriving at a node. However, directed cycles and u-turns are prevented. Once travelers arrive at the destination, their travel routes become the initial travel route and will be updated in subsequent iterations. The randomness of searching direction and the large number of travelers will ensure the diversity of initial route choices, which comprises the choice set on subsequent iterations.

On subsequent iterations, each traveler follows a fixed route chosen at the end of previous iteration. Once arriving at a destination centroid, travelers will enrich the information set with their individual knowledge while benefiting from the pooled knowledge at the same time by exchanging both shortest path and toll information with centroids. Those travelers will also bring that updated information back to their origin and repeat the exchange process. The information exchange mechanism is illustrated by the following graph.

As illustrated in Figure 1, suppose that the traveler originating at node 1 is traveling from node 4 to node 5 . His initial shortest path knowledge is 1-3-4-5. Suppose the shortest path information stored at node 5 is $4-5,3-5,2-3-5$ and 1-2-3-5, respectively from nodes $4,3,2$ and 1 . The comparison starts from the node closest to the current node along the path chain in travelers memory and repeats for each node on this chain until reaching the origin. After comparing the path from node 3 to 5 , the travelers path information is updated to $1-3-5$ since the shortest path for this path segment proposed by the node is shorter than that held by the traveler. Notice that this improvement has also changed 
the shortest path from node 1 to 5 in the travelers memory. Consequently, the node will adopt the path from node 1 proposed by the traveler since 1-3-5 is better than 1-2-3-5. The updated path from node 1 to 5 then becomes part of the travelers shortest path information. This information exchange mechanism will naturally mutate the path chain and generate the most efficient route, sometimes better than all known existing routes. Since nodes store K alternative paths, nodes will insert the path proposed by the visitor in their information pool as long as this path is better than the longest path stored. This information will also be shared by those travelers visiting node 5 at subsequent steps.

After stopping at the destination node, travelers compare their travel route determined at the end of previous iterations and shortest path learned during the currently iteration. The path length is evaluated in dollar values by considering their individual value of time and the toll charged by each link segment. Since travelers have different values of time, the cost of $\mathrm{K}$ alternatives should be reevaluated and sorted for each traveler. If the path suggested by the destination node is better than their current route, the travelers have a probability to switch to the better route that iteration. In general,

$$
P=f(s, b, T)
$$

to apply this model, we choose a specific form:

$$
\left\{\begin{array}{l}
p=s\left(1-e^{-\gamma b}\right) \text { ifb }>\mathrm{T} \\
p=0 \text { otherwise }
\end{array}\right.
$$

where:

b represents the potential benefit by switching routes, which is defined as the time or money saving by choosing route proposed by the destination node instead of sticking to the current route. 
$\mathrm{T}$ is the threshold of benefit perception, which reflects both the incapability to perceive small benefit and the inertia for people to change route.

$\mathrm{s}$ denotes the probability of perceiving an existing better route in a given day, and captures the differentiation in the effectiveness of social networks.

$\gamma$ defines the shape of the probability curve.

ARC simulates the day-to-day route choice behavior of travelers and this probability curve must account for two factors: 1) the probability a traveler perceives this better path once its information is available and 2) the probability a traveler takes this path once it is learned. It should be noted that information spreading costs time and not everyone learns it immediately. Travelers with more effective social networks are more likely to be exposed to such information and thus have a higher probability of learning the better path. Once a new road opens, it takes weeks or even months before the flow reaches at a stable level. Even when people learn a better alternative, route change involves a certain switching cost preventing travelers from changing routes immediately. Or travelers may just resist of changing because of inertia. Considering these factors, this curve should increase as benefits increase and reach some up limit predicted by the probability to learn. Estimation of this curve through survey or other psychological study will enhance the empirical foundation of the model.

Figure 2 illustrated the flow chart of ARC. After travelers choose their routes according to the aforementioned probability, link flow and link travel time will be updated. Consequently, the cost of all possible paths stored both at nodes and travelers will be updated without changing the choice set. Then travelers will follow their new route and repeat the described process until an equilibrium pattern is reached (equilibrium is defined here as link flow variance smaller than a pre-determined threshold $\varepsilon$, we arbitrarily choose $\varepsilon=5$ ). Once this equilibrium is reached, no traveler has the incentive to change the travel route 
according to their behavior rules and available information. Thus a link flow pattern would be reached and could be provided to other models under a more comprehensive framework.

\section{Numerical experiments}

Numerical experiments are conducted on the Sioux Falls network, a widely-used testing network for travel demand modeling. The Sioux Falls network contains 24 nodes and 76 links, and there are 336,400 daily trips according to Bar-Gera (2001). The number of trips between OD pairs has been rounded to integers.

The value of time is an important characteristic of travelers and can be derived from survey data. This study assumes that value of time follows a normal distribution with a mean of $10 \$ /$ hour and a standard error of $2 \$ /$ hour. We then generate our traveler agents by randomly assigning this characteristic subject to the chosen distribution.

Travelers route choice decisions regarding benefits generated from route switching is the behavioral foundation of ARC. It is crucial to decide the overall performance of our traffic assignment model. A route switching probability insensitive to potential benefits leads to premature convergence, which is far-from user equilibrium and generates unrealistic flow distribution among links. In this case, the final flow pattern is primarily determined by the initial route choice of travelers. However, an overly sensitive probability will lead travelers jumping between alternative paths. This flip-flop phenomenon prevents the model from approaching equilibrium and the flow pattern is also unrealistic. A feasible distribution of route choice should follow in between and can be derived from survey or more ideally, from experiment conducted in the field. After testing different parameter combination, we choose the values $(\gamma=1, T=0.1$ and $s=0 . \overline{3}$ uniform for all travelers) ensuring fast convergence and accuracy 
equivalent to UE assignments.

\subsection{Comparison to UE and SUE assignments}

UE assignment (Frank-Wolfe algorithm) and SUE assignment (Dial's algorithm with a $\theta$ of 1 and standard MSA) are common practices in transportation studies. Despite starting from a different modeling philosophy, ARC should be able to generate results consistent with those classical assignment problems with reasonable computing time to validate its applicability in practice. To make these models comparable, we assume the tolls on all links equal zero, keeping other attributes such as capacity and free flow travel time the same as Bar-Gera's network (Bar-Gera, 2001). We also adopted BPR function as our flow-cost function. It takes 35 iterations for ARC to reach equilibrium when simulating 336,400 daily trips on Sioux Falls network. Three models have been coded in Java and applied on an Apple PowerMac G5 2.7GHz.

[Table 1 about here]

Table 1 summarizes the difference in link flow compared to the best-known result proposed by Bar-Gera (according to UE principle) and corresponding running time. This comparison indicates that the macroscopic link flow pattern emerging from the individual decision-making process modeled in this study is very close to the UE link flow pattern. Since both SUE and ARC assume the existence of perception error and probability in route choice, it is not a surprise to observe that ARC generates a flow pattern very similar to that of SUE, which further implies that our assumption in term of route switch probability is reasonable. Therefore, if we incorporate tolls on links and assume static condition, we could expect a link flow pattern close to a UE or SUE assignment based on generalized cost.

Although the running time of ARC looks much longer than that of UE and 
SUE, we must realize that ARC generates much more information at the expense of running time. Actually, we could trace the route choices of individual traveler over time and generate various statistics (e.g. average value of time) of users at different level (such as link, path, zone, and network), which enable study of questions such as equity or market differentiation. Moreover, ARC is more sensitive to the number of travelers than the scale of the network. Noting that the real network contains many more nodes and links but slightly more trips comparing to the simplified SiouxFalls network, ARC shows advantages when applied in practice, which will be demonstrated in the following section.

\subsection{Sensitivity Analysis}

There are several variables in the model that may affect the results. Sensitivity tests are conducted on these variables: size of information set, sensitivity to route switching, and perception threshold. These are discussed in turn for both the Sioux Falls and Chicago networks.

The size of information sets kept on centroids, or the number of alternative paths $(\mathrm{K})$, reflects the diversity of path information available and the extent of peoples information processing capability when making the route choice. Some destinations, such as downtown transportation hubs, are able to provide more choices than others because of their affluence of information. They are also the most frequently visited nodes. This model assumes a uniform size of choice set through the whole network for simplicity and the network performance with different sizes is summarized in Table 2. A larger $\mathrm{K}$ means more alternatives are available when making a route choice and consequently it results in an assignment more comparable to the UE assignment, with other factors controlled. However, more alternatives may lead to more trial-and-error cycles and consequently it may take more iterations to achieve the convergence. As illustrated 
by Table 2, the difference is less significant when $K$ is larger than 4 . We may conclude that four alternatives for each OD is a large enough choice set on the Sioux Falls network. However, whether this conclusion still holds on a larger network is still an open question and needs further tests.

[Table 2 about here]

The changing of route imposes some costs and thus travelers may ignore those routes bringing trivial reduction in travel cost. This reluctance to switch routes is captured by the perception threshold $(\mathrm{T})$ in the model. Outcomes under different threshold choices are summarized in Table 3. A larger threshold means travelers are more reluctant to switch route and the model will consequently converge faster. However, since UE assignment assumes perfect travel cost perception and absolute equilibrium, a larger threshold will also lead to a more significant deviation from UE assignments. Experiments reveal that link flows with a threshold larger than $\mathrm{T}=0.2$ are significantly different than those generated by UE assignment.

[Table 3 about here]

\subsection{Demonstration on larger network}

Although ARC tracks the decisions of individual traveler, it is still tractable on large real network. A demonstration has been conducted on the Chicago Sketch network (CHS), a fairly realistic yet aggregated representation of the Chicago region developed by the Chicago Area Transportation Study (CATS). As an agent-based model, ARC does not deal with fractional demand. Consequently, the original OD table provided by Bar-Gera (2001) has been rounded. In this study, ARC simulates decision-making process of 1.13 million traveler agents on a network with 387 centroids, 933 nodes, and 2950 links. To evaluate the emerging pattern predicted by ARC, the same OD has been assigned using 
Origin Based Algorithm (OBA) developed by Bar-Gera and Boyce (2003) and this assignment is taken as the best known assignment according to Wardrop's principle. All of the three models, ARC, UE (Frank-Wolfe), and SUE (Dial's algorithm with a $\theta$ of 1 and MSA), have been coded in Java and run on the same computer for 100 iterations each. The link flow comparison is summarized in Table 4.

[Table 4 about here]

As indicated in Table 4, ARC generates a flow pattern with a smaller discrepancy compared to the OBA assignment than the alternatives, while UE and SUE have very similar results. Figure 4 and Figure 5 further illustrate that the flow on most links are very consistent with the best-known assignment generated by OBA. This comparison suggests that ARC has very good performance on large networks and this advantage is more significant if we consider the details ARC can provide and the solider behavior foundation. Figure 3 illustrates how maximum link flow difference between consecutive iterations, the convergence criterion adopted in this research, evolves over time for three assignment algorithms. Although the difference compared to the other two algorithm decreases over iterations, ARC converges much faster at the first few iterations and exhibits more stable flow pattern. This is partly due to the behavior assumptions such as information spreading speed and inertia of changing route assumed in ARC, which is more consistent with the real world. Table 5 shows the comparison in computational efficiency on different networks. Although ARC still costs more time compared to UE and SUE, the difference reduces rapidly as the scale of network increases. However, we must indicate that this running time for ARC is based on the simplification of uniform traveler characteristics in order to be comparable with UE or SUE. If we consider heterogeneity in travelers, each iteration of ARC consumes 5.5 minutes and it takes 85 iterations to converge, 
according to a criterion that the maximum relative link flow error between two consecutive iterations should be less than 6 vehicles.

[Table 5 about here]

\section{Application}

The introduction of toll road and price setting is a comprehensively debated topic. One concern about toll roads is the potential inequity. It is intuitive to think that travelers with higher value of time would take toll roads, while poorer travelers select roads without or with fewer tolls. While this is a common argument when people discuss the toll road, the extent of the differentiation on large networks is seldom studied. Because of its capacity to model each individual traveler, ARC has advantages in revealing the relationship between toll rates and traveler differentiation. This paper only illustrates this idea with a simple network, containing only one OD and two alternative routes (A and B). The demand is assumed $2000 \mathrm{veh} / \mathrm{h}$ and each alternative route has a capacity of $800 \mathrm{veh} / \mathrm{h}$. We assume route B is toll free and travelers reactions with different toll rate on route A are studied.

[Table 6 about here]

As illustrated in Table 6, if we charge a higher toll rate on route A, travelers exhibit more significant differentiation: fewer travelers would take route A and those users have a higher value of time. While route B is more congested and serves those travelers with a lower value of time. The extent of differentiation depends on the toll rate charged on route B and the distribution of value of time among travelers. 


\section{Conclusion and Discussion}

Starting from theory about how spatial knowledge is generated, refined and communicated among travelers, the Agent-based Route Choice (ARC) model differs from previous research by generating a route choice set using a behavioral framework. Parameters describe how travelers perceive the available information and make route choice decisions. Upon estimation and calibration of those parameters, a fully behavior based traffic assignment model can be established. ARC simulates how travelers make route choice decisions over time and how the product of those individual decisions evolves into a static (and near-equilibrium) pattern if network conditions hold constant. Numerical experiments suggest that $\mathrm{ARC}$ is realistic, and also feasible even on large networks.

Compared to a traditional UE or SUE assignment, the advantage of ARC relies on its ability to track evolutionary decisions made by heterogeneous travelers, particularly on networks with differentiated levels of service provided by tolls. It is highly likely that travelers with higher value of time will take those paths with higher tolls but lower congestion level, while those less wealthy travelers prefer free public routes. Therefore, this model permits the investigation of issues involving traveler differentiation, such as equity, marketing and toll and investment strategies.

Adopting the evolutionary modeling approach and focusing on behavioral solidity, ARC can account for the presence of exogenous information or nonstatic network condition, in which case the equilibrium modeling paradigm may not apply, since no equilibrium exists in these cases.

More importantly, ARC provides a new modeling platform based on acquisition and processing of spatial information, which is behaviorally sounder than modeling based on shortest path searching. Following the same agentbased modeling paradigm and focusing on the travel behavior, we could revisit 
the problems of travel demand modeling, including residential, job, and nonwork location choice, mode choice, activity generation, and departure time and scheduling. Since we deal with individual travelers, the same traveler agent in also these models can carry identical personal characteristics and preferences. Thus, this modeling framework exhibits the potential to solve the travel demand and routing problems consistently, which has long been proposed by researchers.

\section{References}

Richard Arnott and Marvin Kraus. When are anonymous congestion charges consistent with marginal cost pricing? Journal of Public Economics, 67(1): 45-64, January 1998.

JoseAugusto Azevedo, Maria Emilia O. Santos Costa, Joaquim Joao E. R. Silvestre Madeira, and Ernesto Q. Vieira Martins. An algorithm for the ranking of shortest paths. European Journal of Operational Research, 69(1):97-106, August 1993.

K.W. Axhausen Balmer, M. and K. Nagel. An agent-based demand modeling framework for large scale micro-simulation. In 85th Annual Meeting of Transportation Research Board, Washington D.C., 2006, 2006.

M. Balmer, N. Cetin, Kai Nagel, and B. Raney. Towards truly agent-based traffic and mobility simulations. In Autonomous Agents and Multiagent Systems, 2004. AAMAS 2004. Proceedings of the Third International Joint Conference on, pages 60-67, 2004.

Albert Bandura. Social Learning Theory. Pearson Professional,River, N.J., 1977. 
Hillel Bar-Gera. Transportation network test problems. Ben-Gurion University of Negev, 2001. URL http://www.bgu.ac.il/ bargera/tntp/.

Hillel Bar-Gera and David Boyce. Origin-based algorithms for combined travel forecasting models. Transportation Research Part B: Methodological, 37(5): 405-422, June 2003.

Richard Bellman and Robert Kalaba. On $k$ th best policies. Journal of the Society for Industrial and Applied Mathematics, 8(4):582-588, December 1960. ISSN 0368-4245.

K.J. Button and E.T. Verhoef. Road Pricing, Traffic Congestion and the Environment: Issues of Efficiency and Social Feasibility. Cheltenham: Edward Elgar, 1998.

David Charypar and Kai Nagel. Generating complete all-day activity plans with genetic algorithms. Transportation, 32(4):369-397, July 2005.

Carlos F. Daganzo and Yosef Sheffi. On stochastic models of traffic assignment. Transportation Science, 11(3):253-, August 1977. ISSN 00411655.

T. De La Barra, B. Perez, and J. Anez. Multidimensional path search and assignment. Proceedings of the 21st PTRC Summer Meeting, 363:307-319, 1993.

A. de Palma, G.M. Myers, and Y.Y. Papageorgiou. Rational Choice Under an Imperfect Ability To Choose. The American Economic Review, 84(3): 419-440, 1994.

M. Romero Estache, A. and J. Strong. The long and winding path to private financing and regulation of toll road. Technical report, World Bank Research Working Paper, No. 2387, 2000. 
A Evans. Road congestion pricing? when it is a good policy. Journal of Transportation Economics and Policy, 26:213-243, 1992.

R.G. Golledge and Stimson R.J. Spatial behavior. Guilford Press, New York, 1997.

F.H. Knight. Some fallacies in the interpretation of social cost. Quarterly Journal of Economics, 38:582-606, 1924.

David Levinson. An evolutionary transportation planning model: Structure and application. In Transportation Research Record No. 1493, 1995.

David Levinson. Micro-foundations of congestion and pricing: A game theory perspective. Transportation Research Part A: Policy and Practice, 39(7-9): 691-704, 2005.

Hani S. Mahmassani and Yaser E. Hawas. A hierarchical distributed computational architecture for a centralized real-time dynamic traffic assignment. In TRISTAN III, San Juan, Puerto Rico, 1998.

D. McFadden. Disaggregate behavioral travel demands rum side: A 30-year retrospective. In D. Heshner and J. King, editors, The Leading Edge Of Travel Behavior Research. Pergamon Press, Oxford, 2002.

H. Mohring and M. Harwitz. Highway Benefits: An Analytical Framework. Nothwestern University Press, 1962.

Kai Nagel and Marcus Rickert. Parallel implementation of the transims microsimulation. Parallel Computing, 27(12):1611-1639, November 2001.

R. J. Beckman Nagel, K. and C. L. Barrett. Transims for urban planning. Technical report, Los Alamos National Laboratory Unclassified Report LAUR-98-4389, 1999. 
R.W. Olshavsky. Task complexity and contingent processing in decision making: a replication and extension. Organizational Behavior and Human Performance, 24:300-316, 1979.

J. Bettman Payne, J. and E. Johnson. The Adaptive Decision Maker. Cambridge University Press, NewYork, 1993.

A.C. Pigou. The Economics of Welfare. Macmillan, 1920.

M.S. Ramming. Network Knowledge and Route Choice. PhD thesis, Department of Civil and Environmental Engineering, Massachusetts Institute of Technology, 2002.

Georgina Santos and Laurent Rojey. Distributional impacts of road pricing: The truth behind the myth. Transportation, 31(1):21-42, February 2004.

Karthik K. Srinivasan and Hani S. Mahmassani. Analyzing heterogeneity and unobserved structural effects in route-switching behavior under atis: a dynamic kernel logit formulation. Transportation Research Part B: Methodological, 37(9):793-814, November 2003.

Amos Tversky and Itamar Simonson. Context-dependent preferences. Management Science, 39(10):1179-1189, October 1993. ISSN 00251909.

William S. Vickrey. Pricing in urban and suburban transport. American Economic Review, 53(2):452-, May 1963. ISSN 00028282.

J.G. Wardrop. Some theoretical aspects of road traffic research. In Proceedings of the Institute of Civil Engineers, Pt. II, volume 1, pages 325-378, 1952.

J. Wolpert. The decision process in spatial context. Economics, 69:99-118, 1952. 
Hai Yang and Hai-Jun Huang. The multi-class, multi-criteria traffic network equilibrium and systems optimum problem. Transportation Research Part B: Methodological, 38(1):1-15, January 2004.

Hai Yang and Xiaoning Zhang. Multiclass network toll design problem with social and spatial equity constraints. Journal of Transportation Engineering, 128(5):420-, September 2002. ISSN 0733947X.

Junyi Zhang, Harry Timmermans, Aloys Borgers, and Donggen Wang. Modeling traveler choice behavior using the concepts of relative utility and relative interest. Transportation Research Part B: Methodological, 38(3):215-234, March 2004.

Lei Zhang. Search, Information, Learning, and Knowledge in Travel DecisionMaking. PhD thesis, Department of Civil Engineering, University of Minnesota, 500 Pillsbury Drive S.E., Minneapolis, MN55108, 2006.

Lei Zhang and David Levinson. An agent-based approach to travel demand modeling: An exploratory analysis. In Transportation Research Record: Journal of the Transportation Research Board No.1898, 2004.

Athanasios Ziliaskopoulos. Optimum Path Algorithms on Multidimensional Networks: Analysis, Design, Implementation and Computational Experience. PhD thesis, University of Texas at Austin, 1994.

Xi Zou and David Levinson. A multi-agent congestion and pricing model. Transportmetrica, 2(3):237-249, 2006. 
Table 1: Comparison between link flows on Sioux Falls Network

\begin{tabular}{|c|c|cc|cc|cc|}
\hline & $\begin{array}{l}\text { Best-known } \\
\text { Assginment } \\
(\text { veh } / \text { day })\end{array}$ & UE & \multicolumn{2}{|c|}{ SUE } & \multicolumn{2}{|c|}{ ARC } \\
\hline & & Abs. & Rela. & Abs. & Rela. & Abs. & Rela. \\
\hline mean & 11547 & 40 & $0.30 \%$ & 1080 & $2.10 \%$ & 1043 & $1.60 \%$ \\
\hline maximum & 15333 & 151 & $1 \%$ & 251 & $7 \%$ & 181 & $6.80 \%$ \\
\hline running time & \multicolumn{2}{|c|}{$<2 s$} & \multicolumn{2}{c|}{$<2 s$} & \multicolumn{2}{c|}{$80 s$} \\
\hline
\end{tabular}

Table 2: Comparison between link flows on Sioux Falls Network

\begin{tabular}{|c|c|c|c|}
\hline K & Iterations & Max link flow error $($ veh $)$ & Mean link flow error $($ veh $)$ \\
\hline 1 & 64 & 7943 & 1598 \\
\hline 2 & 82 & 1496 & 329 \\
\hline 4 & 105 & 1212 & 252 \\
\hline 6 & 124 & 1375 & 271 \\
\hline 10 & 119 & 1202 & 268 \\
\hline
\end{tabular}

Table 3: Network performance with different perception thresholds

\begin{tabular}{|l|c|c|c|}
\hline T & Iterations & Max link flow error $($ veh $)$ & Mean link flow error $($ veh $)$ \\
\hline 0.1 & 105 & 1212 & 252 \\
\hline 0.2 & 105 & 1225 & 261 \\
\hline 0.5 & 75 & 1831 & 344 \\
\hline
\end{tabular}

Table 4: Comparison of link flow pattern on Chicago Sketch Network

\begin{tabular}{|c|c|cc|cc|cc|}
\hline & $\begin{array}{l}\text { Best-known } \\
\text { Assginment } \\
\text { veh/day })\end{array}$ & UE (FW) & SUE & \multicolumn{2}{|c|}{ ARC } \\
\hline & & Abs. Rela. & Abs. Rela. & Abs. & Rela. \\
\hline mean & 2383 & 170 & $7 \%$ & 170 & $7 \%$ & 108 & $4.50 \%$ \\
\hline maximum & 22365 & 2906 & $13 \%$ & 2906 & $13 \%$ & 2967 & $13 \%$ \\
\hline running time & & \multicolumn{2}{|c|}{$702 \mathrm{~s}$} & \multicolumn{2}{|c|}{$1038 \mathrm{~s}$} & \multicolumn{2}{|c|}{$4012 \mathrm{~s}$} \\
\hline
\end{tabular}

Table 5: Network Scale and Running Time Comparison

\begin{tabular}{|c|c|c|c|c|ccc|}
\hline & Links & Centroids & Nodes & Trips & \multicolumn{3}{|c|}{ Computing time (sec) } \\
& & & & & UE & SUE & ARC \\
\hline SiouxFalls & 76 & 24 & 24 & 336400 & 2 & 2 & 80 \\
\hline Chicago Sketch & 2950 & 387 & 933 & 1133783 & 702 & 1038 & 4012 \\
\hline Ratio & 38.8 & 16.1 & 38.9 & 3.36 & 351 & 519 & 50 \\
\hline
\end{tabular}


Table 6: Link flows and Users average value of time with different toll rate

\begin{tabular}{|c|cc|lc|ll|}
\hline $\begin{array}{c}\text { Toll on A } \\
(\$)\end{array}$ & \multicolumn{2}{|c|}{ Overall value of time $(v o t)$} & \multicolumn{2}{|l|}{ Route A } & \multicolumn{2}{l|}{ Route B } \\
Mean $(\$ / h)$ & Variance & Flow & vot in $\$$ & Flow & vot in $\$$ \\
\hline 0 & 10 & 5 & 987 & 0.168 & 1013 & 0.171 \\
\hline 0.15 & 10 & 5 & 980 & 0.168 & 1020 & 0.171 \\
\hline 0.3 & 10 & 5 & 932 & 0.169 & 1068 & 0.17 \\
\hline 0.5 & 10 & 5 & 786 & 0.175 & 1214 & 0.166 \\
\hline 1 & 10 & 5 & 591 & 0.184 & 1409 & 0.163 \\
\hline 2 & 10 & 5 & 321 & 0.229 & 1679 & 0.158 \\
\hline 2 & 20 & 5 & 535 & 0.345 & 1465 & 0.332 \\
\hline
\end{tabular}



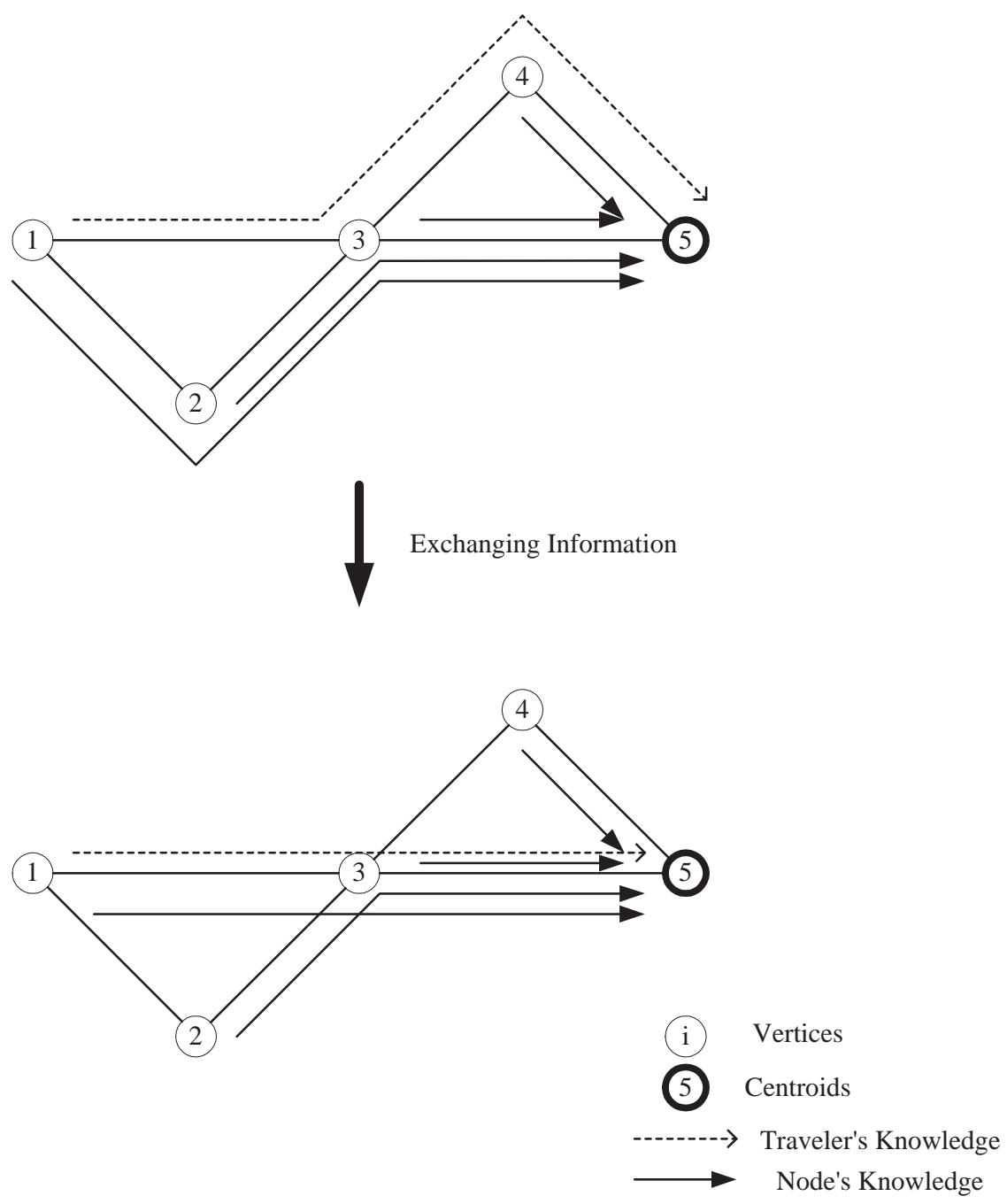

Figure 1: Information Exchange Mechanism 


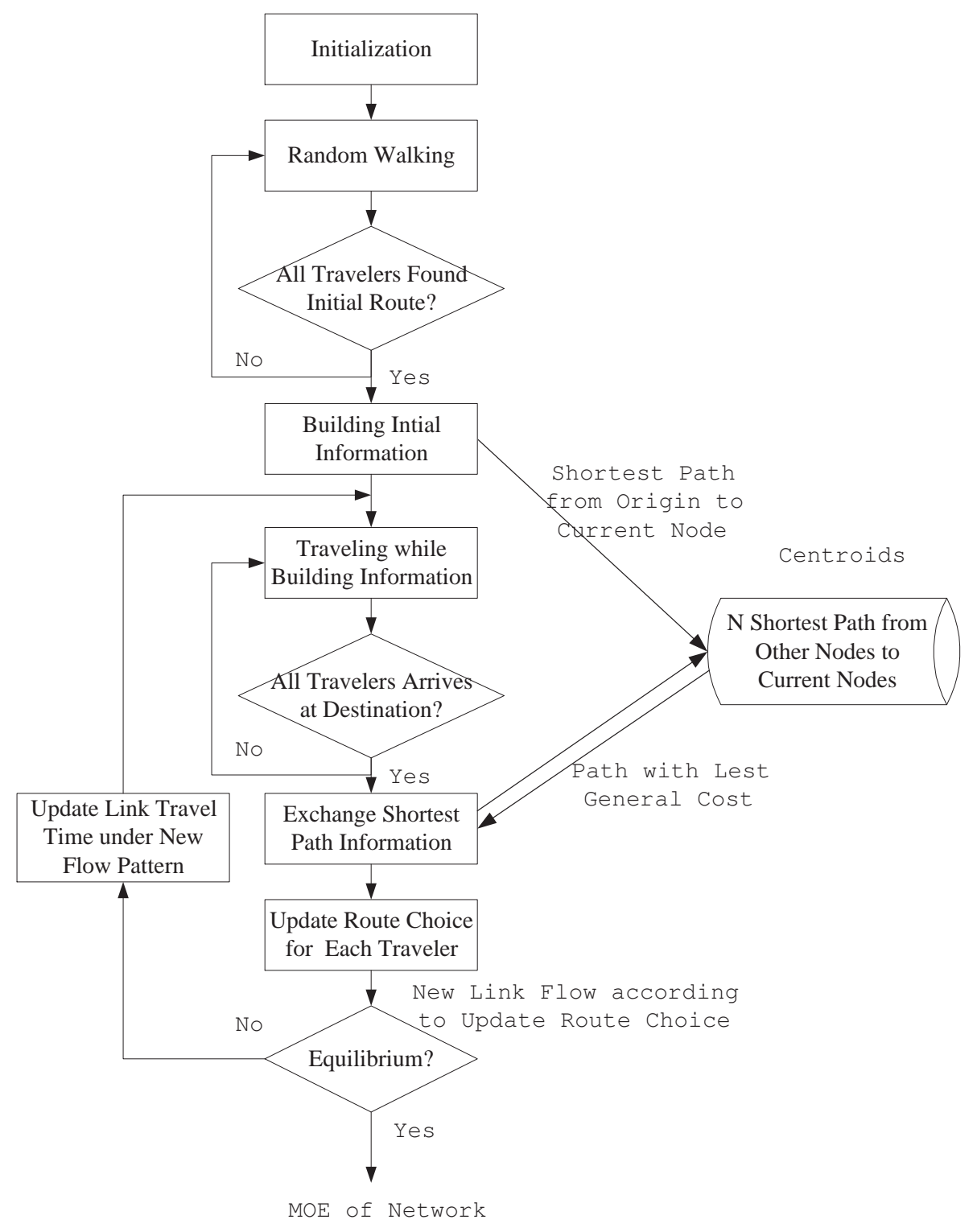

Figure 2: Flow Chart of Agent-based Route Choice Model 


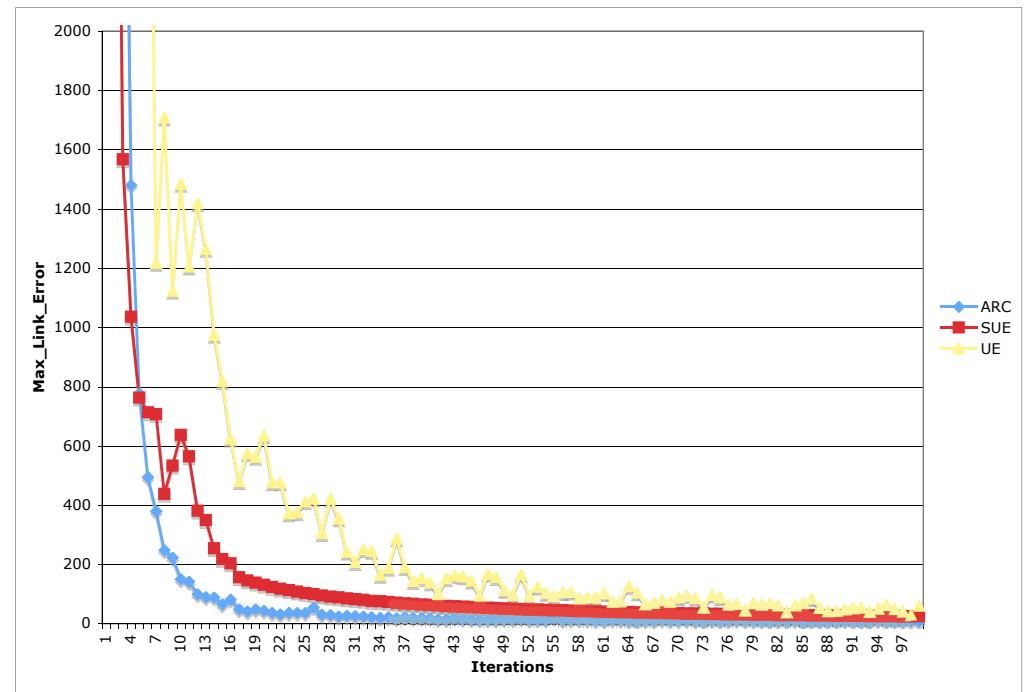

Figure 3: Convergence speed comparison among ARC, UE, SUE on CHS 


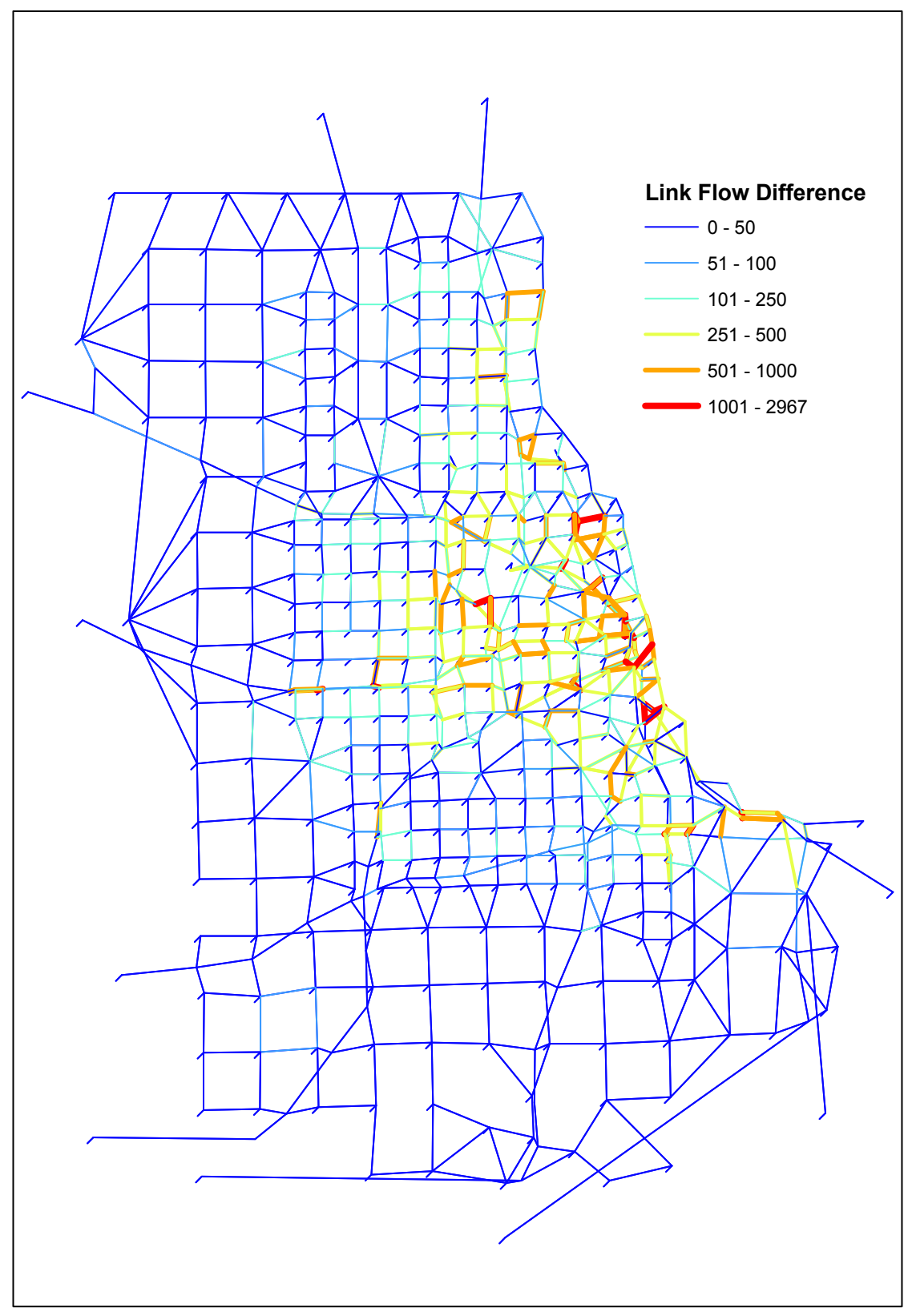

Figure 4: Error in Link Flow between ARC and OBA assignment on CHS 


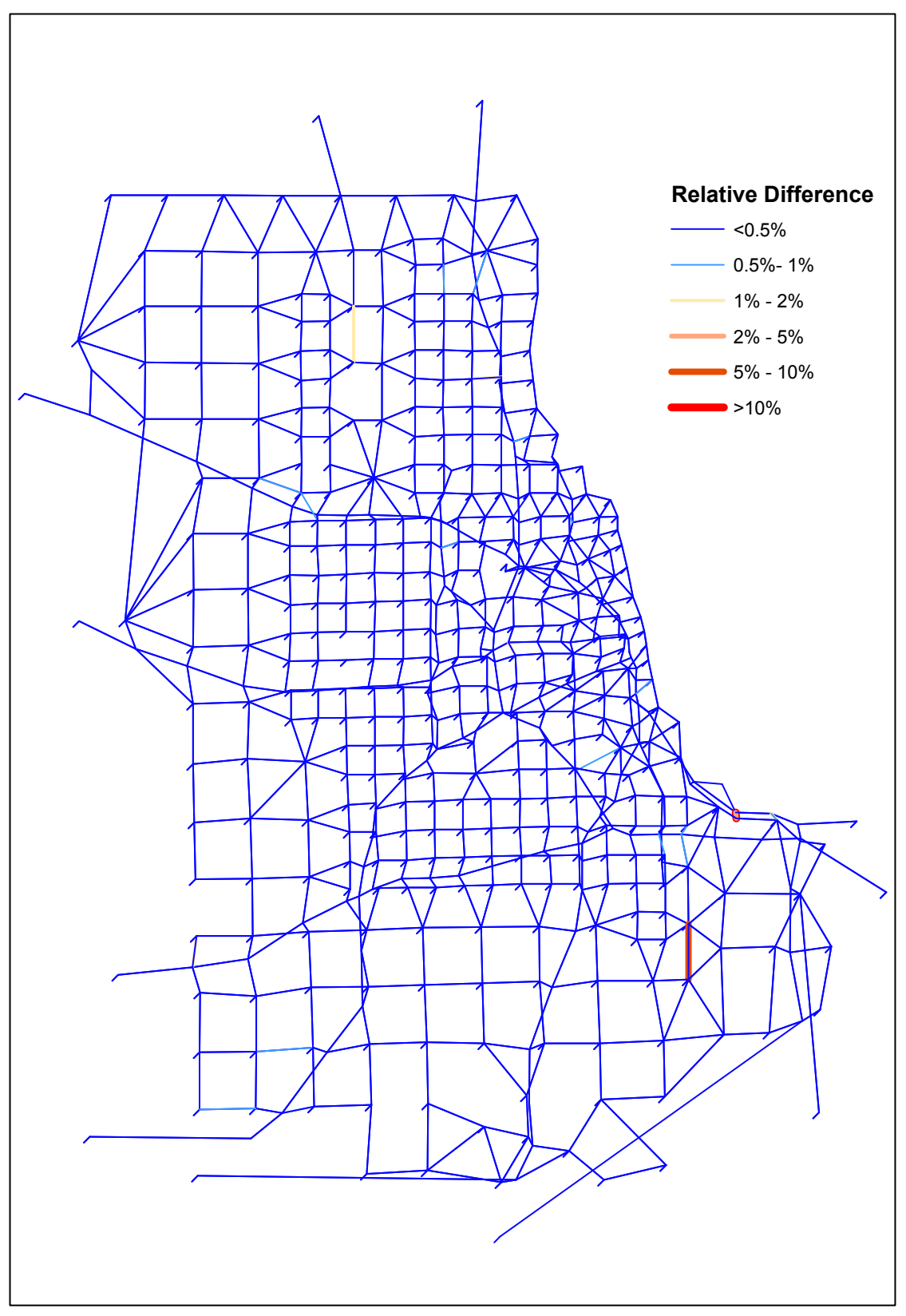

Figure 5: Relative Error in Link Flow between ARC and OBA assignment on CHS 